\title{
Cucurbitacin B induces autophagy and apoptosis by suppressing CIP2A/PP2A/mTORC1 signaling axis in human cisplatin resistant gastric cancer cells
}

\author{
XUEWEN LIU ${ }^{1,3^{*}}$, CHAO DUAN ${ }^{1 *}$, JUANLI JI ${ }^{1}$, TE ZHANG $^{1}$, XIAONING YUAN ${ }^{1}$, YUNFEI ZHANG ${ }^{1}$, \\ WENJING MA ${ }^{1}$, JINGYUAN YANG ${ }^{2}$, LINSEN YANG ${ }^{2}$, ZHIGUO JIANG ${ }^{2}$, HUILIANG YU ${ }^{2}$ and YING LIU ${ }^{1,3}$ \\ ${ }^{1}$ Laboratory of Molecular Target Therapy of Cancer, Institute of Basic Medical Sciences, Hubei University of Medicine, \\ Shiyan, Hubei 442000; ${ }^{2}$ Hubei Province Key Laboratory of Conservation Biology for Shennongjia Golden Monkey, \\ Shennongjia National Nature Reserve, Shennongjia Forestry Region, Shennongjia, Hubei 442400; ${ }^{3}$ School of Basic \\ Medical Sciences, Hubei University of Medicine, Shiyan, Hubei 442000, P.R. China
}

Received November 13, 2016; Accepted May 8, 2017

DOI: $10.3892 /$ or.2017.5648

\begin{abstract}
Cancerous inhibitor of protein phosphatase 2A (CIP2A) is a human oncoprotein that is overexpressed in multiple kinds of tumors including gastric cancer (GC). Mammalian target of rapamycin complex 1 (mTORC1) overactivation is detected in GC and many other cancers. Previous study found that CIP2A/mTORC1 controls cell growth and autophagy through direct association. CIP2A plays an 'oncogenic nexus' in several cancer types to participate in the tumorigenic transformation and chemoresistance. In the present study, we investigated whether Cucurbitacin $\mathrm{B}(\mathrm{CuB})$, a natural compound found in Cucurbitaceae, can be used in cisplatin (DDP)-resistant human GC cell line SGC7901/DDP. Results demonstrated that CuB treatment significantly suppressed SGC7901/DDP cell proliferation, induced caspase-dependent apoptosis, and autophagy. The activation of autophagy was mediated through $\mathrm{CuB}$-induced inhibition of mTORC1. Furthermore, $\mathrm{CuB}$ inhibited mTORC1 via the activation of protein phosphatase 2A (PP2A) which is mediated by CIP2A inhibition. These findings indicated that $\mathrm{CuB}$ can inhibit the proliferation, induce caspasedependent apoptosis, and autophagy of SGC7901/DDP cells by suppressing CIP2A/PP2A/mTORC1 signaling axis. Thus, $\mathrm{CuB}$ may be a novel effective candidate to treat DDP-resistant human GC cells.
\end{abstract}

Correspondence to: Dr Ying Liu, Laboratory of Molecular Target Therapy of Cancer, Institute of Basic Medical Sciences, Hubei University of Medicine, Shiyan, Hubei 442000, P.R. China

E-mail: ying_liu1002@163.com

"Contributed equally

Key words: Cucurbitacin B, gastric cancer, CIP2A, mTORC1, autophagy, apoptosis

\section{Introduction}

Gastric cancer (GC) is a type of common and fatal digestive malignant disease worldwide. Despite recent advances in diagnosis and treatment as well as declining incidence in some developed countries, it remains a major cause of cancerrelated deaths in Eastern Asia (including China, Japan, and South Korea) $(1,2)$. GC in early stages is curable by using endoscopic procedures. In China, approximately two-thirds of patients develop advanced or metastatic disease, and $>50 \%$ have recurrent disease following curative surgery. Effective therapeutic approaches are still limited (3). Systematic chemotherapy is an extremely important therapeutic strategy for advanced GC. Among the patients, many are resistant to chemotherapy agents, including cisplatin (DDP), adriamycin, and 5-fluorouracil. Multidrug resistance (MDR) is largely responsible for ineffective chemotherapy (4). The mechanism of MDR remains unclear. Increased expression of MDR-associated protein (MRP), P-glycoprotein (P-gp), increased DNA damage repair, cell cycle arrest, and resistance of tumor cells to apoptosis are the mechanisms that might account for MDR (5). A large number of studies have shown that the dysfunction of signaling pathways in cancer cells is the major reason for tumorigenesis and metastasis (6). Therefore, finding novel therapeutic strategies and key target molecules for reversing resistance and diminishing the side effects of chemotherapy agents are the main goals of drug resistance cancer treatment protocol.

Mammalian target of rapamycin complex 1 (mTORC1), a complex composed of mTOR, Raptor and mSIN1, controls protein translation, cell growth, and metabolism (7). mTORC1 over-activation is detected in GC and many other cancers (7-11). mTORC1 activation could induce transcription of a number of key oncoproteins and MDR-associated proteins including cyclin D1 and hypoxia-inducible factor $1 \alpha$ $(\mathrm{HIF}-1 \alpha)(12)$. Activation of mTORC1 results in phosphorylation of important effectors [i.e., ribosomal protein S6 kinase (S6K) and eukaryotic translation initiation factor 4E-binding protein 1 (4E-BP1)] involved in cancer progression and 
apoptosis resistance. In addition, mTORC1 is also the major negative regulator of autophagy (7). Therefore, targeting mTORC1 may be an attractive therapeutic strategy for cancer drug resistance treatment.

The activation of mTORC1 is induced by numerous oncoproteins and mitogenic factors via the class I phosphoinositide3 kinase (PI3K)/Akt pathway (13). Cancerous inhibitor of protein phosphatase 2A (CIP2A) is a human oncoprotein that stabilizes c-Myc and activates Akt by inhibiting protein phosphatase 2A (PP2A)-mediated dephosphorylation of c-Myc and Akt (14). CIP2A can associate with mTORC1 directly and act as an allosteric inhibitor of mTORC1-associated PP2A, thereby enhancing mTORC1dependent growth signaling and inhibiting autophagy (7). An increased expression of CIP2A protein was found in GC and several other cancers (14-18). In 2015, Zhang et al reported that CIP2A expression is associated with DDP resistance (19). Thus, effective and discerning CIP2A-mTORC1 axis inhibitors would be beneficial for therapy to DDP resistance and induce autophagy in GC.

Cucurbitacin $\mathrm{B}(\mathrm{CuB})$ is a natural product that is extensively distributed in medicinal plants Cucurbitaceae family is shown to inhibit the growth of numerous human cancer cell lines such as colon, breast, leukemia, pancreatic hepatic, and glioblastoma (20-22). The effect of CuB on DDP-resistant GC cells previously has not been evaluated. The aim of this work was to investigate antitumor effects and possible mechanisms of $\mathrm{CuB}$ on DDP resistant human GC cells.

\section{Materials and methods}

Reagents. CuB with a purity of up to $98 \%$ was purchased from Shanghai Yuanye Bio-Technology Co., Ltd. CuB was dissolved in dimethyl sulfoxide (DMSO; Sigma-Aldrich, St. Louis, MO, USA) at a stock solution of $40 \mathrm{mM}$ and stored at $-20^{\circ} \mathrm{C}$. DDP and 3-methyladenine (3-MA, \#M9281) were purchased from Sigma-Aldrich.

Cell culture. Human DDP-resistant gastric cancer cell line SGC7901/DDP and human GC cell line SGC7901 were purchased from the Shanghai Institute of Biochemistry and Cell Biology, Chinese Academy of Sciences (Shanghai, China). SGC7901/DDP cells were grown in RPMI-1640 (Gibco; Thermo Fisher Scientific, Inc., Waltham, MA, USA) with fetal bovine serum (FBS; HyClone; GE Healthcare Life Sciences, Chalfont, UK), $500 \mathrm{ng} / \mathrm{ml}$ DDP, and antibiotics and incubated in a humidified atmosphere without $\mathrm{CO}_{2}$ at $37^{\circ} \mathrm{C}$. SGC7901 cells were grown in Dulbecco's modified Eagle's medium (DMEM; Gibco; Thermo Fisher Scientific, Inc.) with FBS and antibiotics and incubated in a humidified atmosphere with $\mathrm{CO}_{2}$ at $37^{\circ} \mathrm{C}$.

Cytotoxic assay and cell viability. Cells were seeded into 96-well plate and pre-cultured for $24 \mathrm{~h}$, then treated with $\mathrm{CuB}$ for $24 \mathrm{~h}$. Cell cytotoxicity was determined by MTT assay. The absorbance was measured at $490 \mathrm{~nm}$ by automated microplated reader (BioTek Instruments, Inc., Winooski, VT, USA), and the inhibition rate was calculated as followed: Inhibition rate $(\%)=($ average A490 of the control group - average A490 of the experimental group) / (average A490 of the control group - average A490 of the blank group) x100\%. Cell viability was estimated by trypan blue dye exclusion $(23,24)$.

Soft-agar colony formation assay. Cells were suspended in $1 \mathrm{ml}$ of RPMI-1640 containing $0.3 \%$ low-melting-point agarose (Amresco, Cleveland, OH, USA) and 10\% FBS, and plated on a bottom layer containing $0.6 \%$ agarose and $10 \%$ FBS in 6-well plate in triplicate. After 2 weeks, plates were stained with $0.2 \%$ gentian violet and the colonies were counted under a light microscope (IX70; Olympus Corp., Tokyo, Japan) (25).

Apoptosis determination by DAPI staining. Approximately $2 \times 10^{5}$ cells/well of cells in a 12 -well plate was treated with $\mathrm{CuB}$ for $24 \mathrm{~h}$. Then cells in each treatment and control were stained by DAPI and examined and photographed under fluorescence microscopy (IX73; Olympus Corp.) as described elsewhere (26).

Western blotting. Cell pellets were lysed in RIPA buffer containing $50 \mathrm{mM}$ Tris $\mathrm{pH} 8.0,150 \mathrm{mM} \mathrm{NaCl}, 0.1 \%$ SDS, $0.5 \%$ deoxycholate, $1 \% \mathrm{NP}-40,1 \mathrm{mM}$ DTT, $1 \mathrm{mM} \mathrm{NaF}$, $1 \mathrm{mM}$ sodium vanadate, $1 \mathrm{mM}$ PMSF (Sigma-Aldrich; Merck Millipore, Darmstadt, Germany), and 1\% protease inhibitors cocktail (Merck Millipore). Lysates were normalized for total protein $(25 \mu \mathrm{g})$ and loaded on $8-12 \%$ sodium dodecyl sulfate polyacrylamide gel, electrophoresed, and transferred to a PVDF membrane (Merck \& Co., Inc., Kenilworth, NJ, USA), followed by blocking with $5 \%$ skimmed milk at room temperature for $1 \mathrm{~h}$. The membrane was incubated with primary antibodies overnight at $4^{\circ} \mathrm{C}$ and rinsed with Tris-buffered saline with Tween-20.

The primary antibodies used were anti-MRP1 (ratio of 1:500 dilution; catalog no. sc-13960), anti-CIP2A (1:500; catalog no. sc-80662), anti-phospho-Akt (S473) (1:500; catalog no. sc-7985), anti-Akt (1:500; catalog no. sc-8312), (Santa Cruz Biotechnology, Santa Cruz, CA, USA), anti-caspase-9 (1:1000; catalog no.9508), anti-caspase-3 (1:1,000; catalog no.9662), antiPARP (1:1,000; catalog no. 9542), anti-PP2A (1:1,000; catalog no. 2038), anti-LC3B (1:1,000; catalog no. 2775), anti-HIF $\alpha$ (1:1,000; catalog no. 5537), anti-p4E-BP1 (Thr37/46) (1:1,000; catalog no. 2855), anti-mTOR (1:1,000; catalog no. 2983), antipmTOR (Ser2448) (1:1,000; catalog no. 5536), anti-P70S6K (1:1,000; catalog no. 9202), anti-pP70S6K (Thr389) (1:1,000; catalog no. 92775) (Cell Signaling Technology, Danvers, MA, USA), anti-Beclin1 (1:2,000; catalog no. 11306-1-AP), anti4E-BP1 (1:2,000; catalog no. 60624-1-Ig) (Proteintech Group, Inc., Rosemont, USA), anti-P-gp (1:2,000; catalog no. ab170904) (Abcam, Cambridge, UK), and anti-GAPDH (1:5,000; catalog no. M20006; Abmart, Shanghai, China).

The blots were then washed, and incubated with horseradish peroxidase (HRP)-conjugated secondary antibody (1:10,000; catalog no. E030120-01 and E030110-01; EarthOx, LLC, San Francisco, CA, USA) at room temperature for $1.5 \mathrm{~h}$. Detection was performed by using a SuperSignal ${ }^{\circledR}$ West Pico Trial kit (catalog no. QA210131; Pierce Biotechnology, Inc., Rockford, IL, USA) (25). The defined sections of the film were scanned for image capture and quantification using Adobe Photoshop software (CS4, Adobe Systems Inc., San Jose, CA, USA) and ImageJ software (National Institutes of Health, Bethesda, MD, USA). 
A

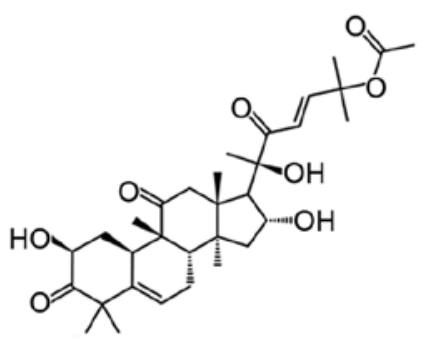

B

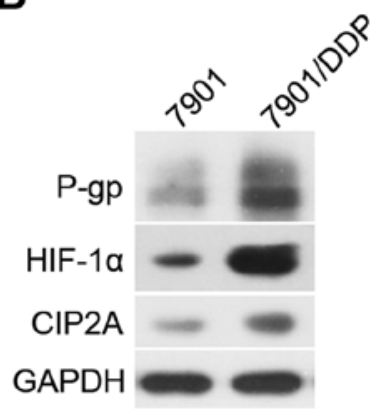

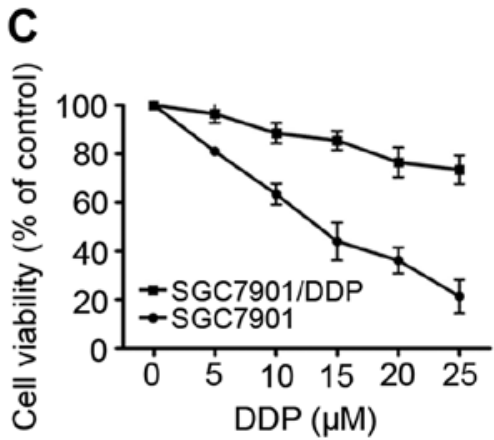

Figure 1. Chemical structure of Cucurbitacin B (CuB) and characterization of SGC7901 and SGC7901/DDP cells. (A) Chemical structure of CuB. (B) Western blotting analysis showing protein expression in SGC7901 and SGC7901/DDP cells using the antibodies indicated. (C) Cell viability was determined by MTT assay after DDP treatment in SGC7901 and SGC7901/DDP cells for $24 \mathrm{~h}$.

Autophagy assays. The cells were transfected with pQCXIPGFP-LC3 plasmid using the Lipofectamine 3000 (Invitrogen; Thermo Fisher Scientific, Inc.) according to the recommended protocol by the manufacturer and then fixed in $4 \%$ paraformaldehyde. The percentage of cells with fluorescent dots representing GFP-LC3 translocation was counted by Olympus confocal laser scanning microscope.

PP2A activity assay. PP2A immunoprecipitation phosphatase assay kit (Upstate, Temecula, CA, USA) was used to measure phosphate release as an index of phosphatase activity according to the manufacturer's instructions. Briefly, $100 \mu \mathrm{g}$ protein isolated from cells was incubated with $4 \mu \mathrm{g}$ anti-PP2A monoclonal antibody overnight. Protein A agarose beads $(40 \mu \mathrm{l})$ were added and the mixture was incubated at $4^{\circ} \mathrm{C}$ for $2 \mathrm{~h}$. Subsequently, the beads were collected and washed three times with $700 \mu \mathrm{l}$ of ice-cold TBS and one time with $500 \mu \mathrm{l}$ Ser/Thr Assay Buffer. The beads were further incubated with $750 \mathrm{mM}$ phosphopeptide in assay buffer for $10 \mathrm{~min}$ at $30^{\circ} \mathrm{C}$ with constant agitation. $100 \mu 1$ of Malachite Green Phosphate Detection Solution was added and the absorbance at $650 \mathrm{~nm}$ was measured on a microplate reader (27).

Transfection of DNA and siRNA. The pOTENT-1-CIP2A expression plasmid was purchased from Youbio Co. (Changcha, China). Transfection of the pOTENT-1-CIP2A plasmid into GC cells were carried out using Lipofectamine 3000 transfection reagent (Invitrogen; Thermo Fisher Scientific, Inc.) following the manufacturer's protocol. Two siRNAs targeting CIP2A were designed and synthesized by Shanghai GenePharma Co., referred to as siRNA1 and siRNA2. The siRNA sequences were as follows: 5'-CUGUGGUUGUGUUUGCACUTT-3' (CIP2A siRNA1), 5'-ACCAUUGAUAUCCUUAGAATT-3' (CIP2A siRNA2), 5'-UUCUCCGAACGUGUCACGUTT-3' [negative control (NC) siRNA].

Using Lipofectamine 2000 (Invitrogen; Thermo Fisher Scientific, Inc.) according to the manufacturer's protocol, SGC7901/DDP cells were transfected with $100 \mathrm{nM}$ siRNA. At $48 \mathrm{~h}$ after transfection, the cells were then harvested for western blotting, and cell viability (28).

Statistical analysis. All experiments were repeated at least three times and the data are presented as the mean \pm SD unless noted otherwise. Differences between data groups were evalu-
Table I. $\mathrm{IC}_{50}$ of $\mathrm{CuB}$ on gastric cancer cell lines.

\begin{tabular}{lcc}
\hline Cell lines & SGC7901 & SGC7901/DDP \\
\hline $\mathrm{IC}_{50}(\mathrm{nM})$ & $216.70 \pm 34.23$ & $170.25 \pm 26.78$ \\
\hline
\end{tabular}

The cells were treated with $\mathrm{CuB}$ at various concentrations for $24 \mathrm{~h}$, the cell cytotoxicity was analyzed by MTT assay, and the $\mathrm{IC}_{50}$ was calculated using CalcuSyn (version 2.0, Biosoft, Cambridge, UK). Values shown are means \pm SD of quadruplicate determination.

ated for significance using Student's t-test of unpaired data or one way analysis of variance and Bonferroni post hoc test. P-values $<0.05$ indicate statistical significance.

\section{Results}

Chemical structure of $C u B$ and characterization of SGC7901 and SGC7901/DDP GC cells. The chemical structure of $\mathrm{CuB}$ is shown in Fig. 1A. Firstly, the P-gp, HIF-1 $\alpha$, and CIP2A expression was compared between SGC7901 and SGC7901/DDP cells, which confirmed P-gp, HIF-1 $\alpha$, and CIP2A were overexpressed in SGC7901/DDP cells (Fig. 1B). SGC7901 and SGC7901/DDP cells were exposed to various concentrations of DDP $(2.5-80 \mu \mathrm{M})$ for $24 \mathrm{~h}$. The halfmaximal inhibitory concentration $\left(\mathrm{IC}_{50}\right)$ of DDP against SGC7901 cells is $6.2 \mu \mathrm{M}$. while $\mathrm{IC}_{50}$ of DDP against SGC7901/DDP cells is $37.78 \mu \mathrm{M}$. As shown in Fig. 1C, the DDP cytotoxicity was higher in SGC7901 cells than in SGC7901/DDP cells.

Effects of CuB on SGC7901 and SGC7901/DDP GC cells. SGC7901 and SGC7901/DDP cells were seeded in 96-well plates for $24 \mathrm{~h}, 48 \mathrm{~h}$ and then treated with different concentrations of $\mathrm{CuB}$ and DDP (Fig. 2A-C). After 24 or $48 \mathrm{~h}$, the cell viability was evaluated by the MTT assay according to the manual. Absorbance at $490 \mathrm{~nm}$ was measured on an automated microplate reader. We found that $\mathrm{CuB}$ had moderate cytotoxicity to SGC7901 and SGC7901/DDP cells with an $\mathrm{IC}_{50}$ of 216.70 and $170.25 \mathrm{nM}$ (Table I). By trypan blue exclusion assay, we found that $\mathrm{CuB}$ rapidly reduced viable SGC7901/DDP cells (Fig. 2D) in a dose- and time-dependent manner. We 
A

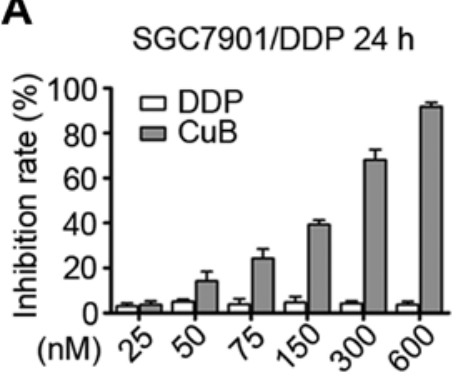

C

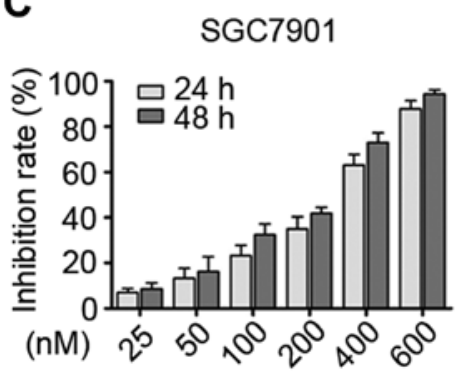

E

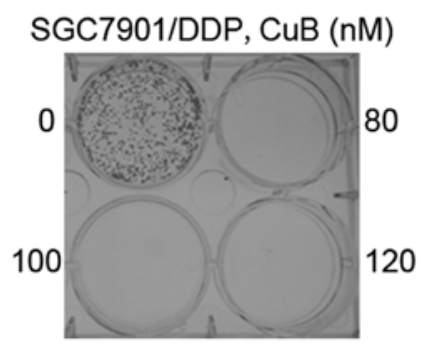

B

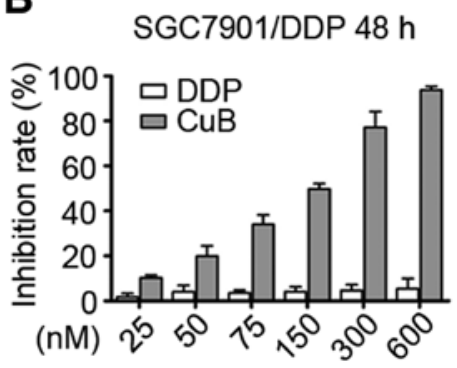

D
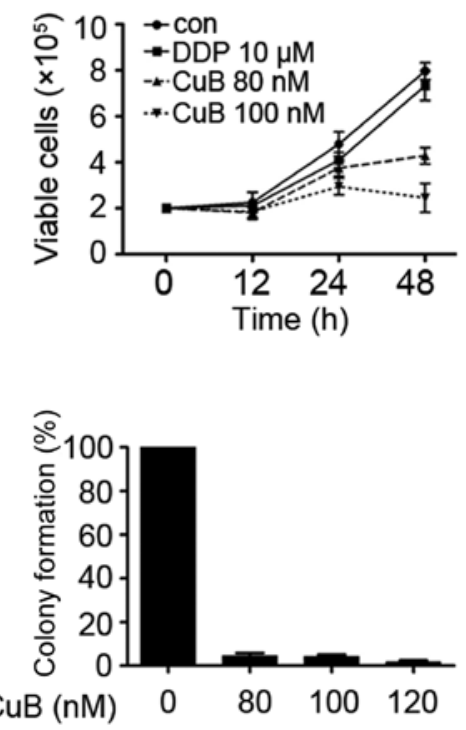

Figure 2. Effect of Cucurbitacin B (CuB) on SGC7901 and SGC7901/DDP GC cells. (A-C) The inhibitory effects of CuB on SGC7901 and SGC7901/DDP GC cells analyzed by MTT assay. (D) Inhibitory effects of CuB and DDP on cell viability of SGC7901/DDP cells assayed by trypan blue exclusion assay. (E) The colony formation assays of SGC7901/DDP cells treated with CuB and DDP at the indicated concentration.

investigated the $\mathrm{CuB}$ effect on cell colony formation activity, and the results showed that $\mathrm{CuB}$ significantly inhibited the clonogenic ability of SGC7901/DDP (Fig. 2E). These results suggested that $\mathrm{CuB}$ inhibited the anchorage-dependent (cell proliferation) and anchorage-independent (colony formation) growth of SGC7901/DDP cells.

CuB induces apoptosis and autophagic death of SGC7901/ DDP GC cells. To investigate whether CuB induced apoptosis in SGC7901/DDP GC cells, microscopy with DAPI staining was carried out. As shown in Fig. 3A, cytoplasmic shrinkage occurred after treatment with $100 \mathrm{nM} \mathrm{CuB}$, supporting that apoptosis was induced. To determine which cell death pathways was induced by CuB, SGC7901/DDP cells were treated with increasing concentrations of $\mathrm{CuB}$ for $24 \mathrm{~h}$. Western blotting results showed that PARP cleavage, as well as a significant dose-dependent decrease in pro-caspases-3 and -9 (pro-casp-3 and pro-casp-9), were detected in CuB-treated cells (Fig. 3A and B). These results indicate that $\mathrm{CuB}$ induces cell death via caspase-dependent apoptosis.

Autophagy is a lysosomal degradation process for cytoplasmic constituents during the stress condition. To assess whether autophagy is also involved in $\mathrm{CuB}$-induced cell death, we subsequently evaluated the expression level of LC3 II and
Beclin1 of cells treated with CuB. A marked increase of LC3 II and Beclin1 was observed in $\mathrm{CuB}$ treated cells (Fig. 3C). When autophagy is initiated, LC3 is cut on the C-terminal and produces LC3 II protein, which is then transferred on the autophagosomes (LC3-positive vesicle) (29). To detect the autophagosome formation, the fluorescent autophagy marker GFP-LC3 plasmid was transfected into SGC7901/DDP cells which were then treated with $\mathrm{CuB}$ for $24 \mathrm{~h}$, followed by confocal microscopy assessment. We showed that while control cells displayed a diffuse staining, SGC7901/DDP cells upon $\mathrm{CuB}$ exhibited a speckled fluorescent staining pattern, indicating the redistribution of LC3 to autophagosomes (Fig. 3D). Autophagy has been reported to play contradictory roles in tumor progression or suppression (30). To demonstrate $\mathrm{CuB}$ induced autophagic cell death, $\mathrm{CuB}$ and autophagy inhibitor 3-MA was combined to treat SGC7901/DDP cells (Fig. 3E). Our results showed that 3-MA significantly reversed the cell proliferation inhibited by $\mathrm{CuB}$. Taken together, these findings suggest that $\mathrm{CuB}$ induces caspase-dependent apoptosis and autophagic death of SGC7901/DDP cells.

$C u B$ influences the expression of $P-g p, H I F-1 \alpha$ and inhibits CIP2A/PP2A/mTORC1 signaling axis in SGC7901/DDP cells. We detected expression levels of MDR related factors 
A

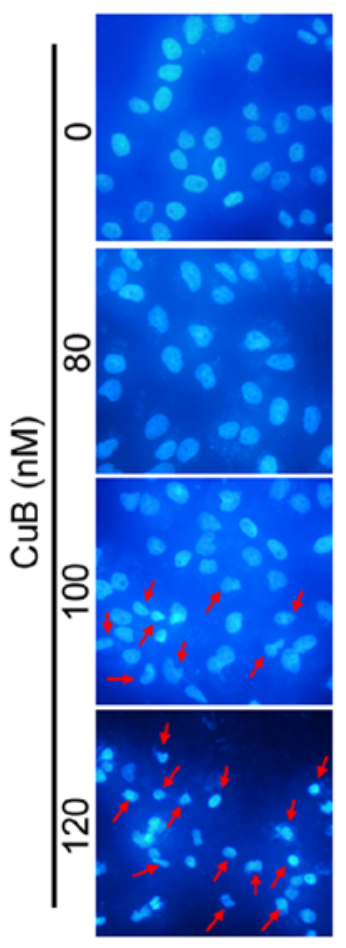

B

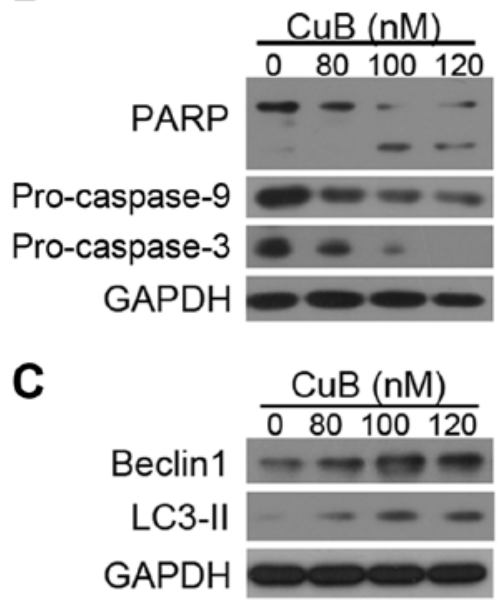

E

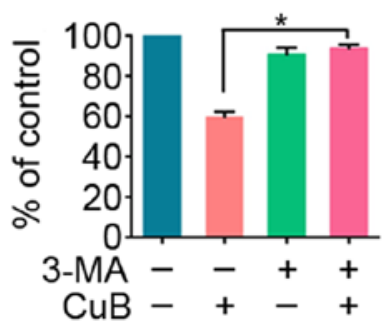

D

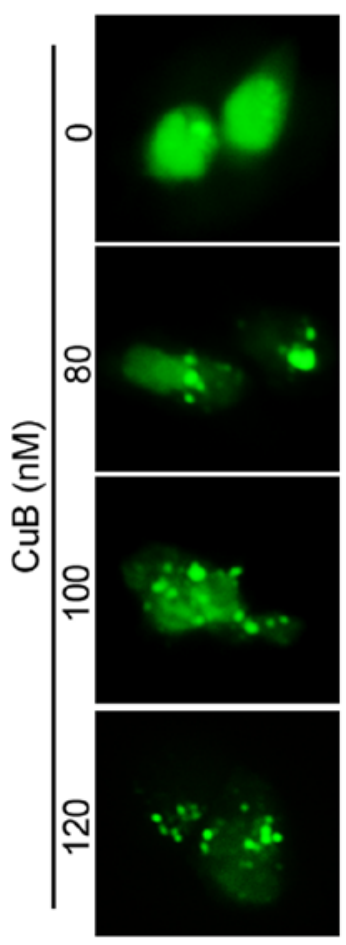

Figure 3. Cucurbitacin B (CuB) induces apoptosis and autophagic death of SGC7901/DDP GC cells. (A) SGC7901/DDP cells were incubated with various doses of $\mathrm{CuB}$ for $24 \mathrm{~h}$. Cells were examined by DAPI staining. (B and C) SGC7901/DDP cells were treated with increasing concentrations of CuB for $24 \mathrm{~h}$. Western blotting was conducted using antibodies indicated. (D) SGC7901/DDP cells transfected with pQCXIP-GFP-LC3 plasmid were treated with increasing concentrations of $\mathrm{CuB}$ for $24 \mathrm{~h}$, and assessed by immunofluorescence analyses. (E) SGC7901/DDP cells were treated with CuB (120 nM) and/or 3-MA (1 mM) for $24 \mathrm{~h}$ and analyzed by MTT assay. ${ }^{*} \mathrm{P}<0.05$.

A

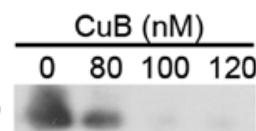

HIF-1a

GAPDH

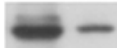

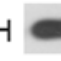

C

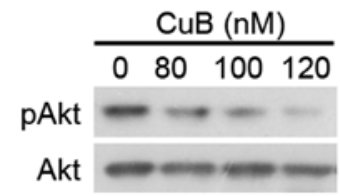

D

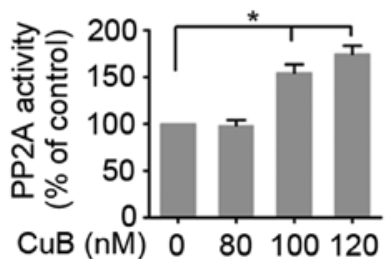

B

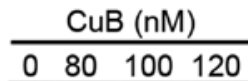

p4E-BP1

4E-BP1

pP70S6K

P70S6K

pmTOR

mTOR

GAPDH

E

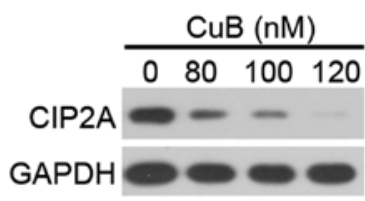

Figure 4. Cucurbitacin B (CuB) influences the expression of P-gp, HIF-1 $\alpha$ and inhibits CIP2A/PP2A/mTORC1 signaling axis in SGC7901/DDP cells. (A-C) SGC7901/DDP cells were treated with increasing concentrations of CuB for $24 \mathrm{~h}$. Western blotting was conducted using the antibodies indicated. (D) SGC7901/DDP cells were treated with increasing concentrations of CuB for $24 \mathrm{~h}$. PP2A activity was measured by PP2A immunoprecipitation phosphatase assay. ${ }^{\mathrm{P}}<0.05$. (E) SGC7901/DDP cells were treated with increasing concentrations of CuB for $24 \mathrm{~h}$.

P-gp, and HIF-1 $\alpha$ by western blotting analysis (Fig. 4A). The results indicated that P-gp, and HIF-1 $\alpha$ expression of
SGC7901/DDP cells were downregulated by treatment with increasing concentration of $\mathrm{CuB}$. The decreased expression 
A

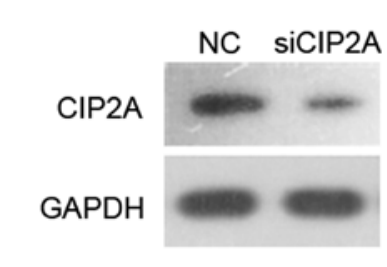

C

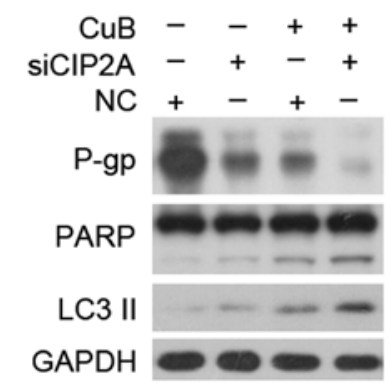

E

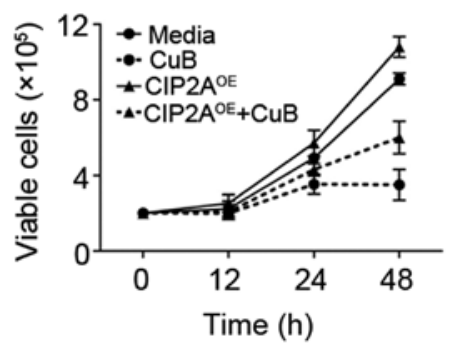

B
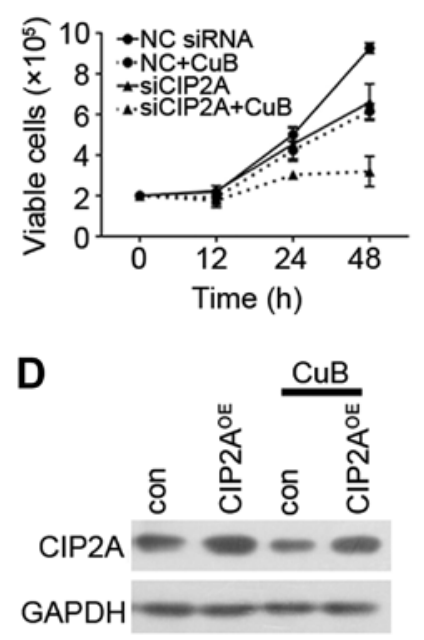

$\mathbf{F}$

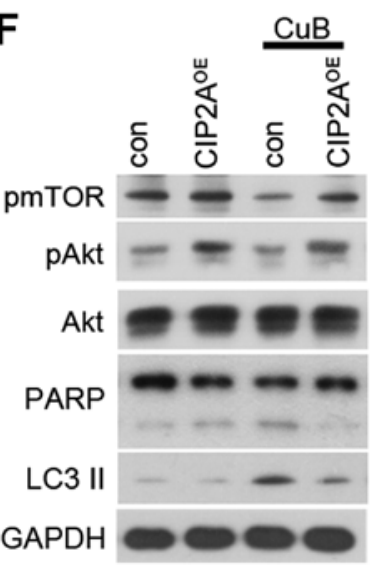

Figure 5. CIP2A is essential for Cucurbitacin B (CuB)-induced proliferation, autophagy, and apoptosis. (A) SGC7901/DDP cells were transfected with 100 nM CIP2A-specific siRNA or NC siRNA for $48 \mathrm{~h}$. Cells were harvested for western blotting analyses. (B) SGC7901/DDP cells were transfected with CIP2Aspecific siRNA or NC siRNA, followed by treatment with $\mathrm{CuB}(80 \mathrm{nM})$ for indicated times, and analyzed by trypan blue exclusion assay. ${ }^{*} \mathrm{P}<0.05$. (C) SGC7901/DDP cells were transfected with CIP2A-specific siRNA or NC siRNA, followed by treatment with CuB (80 nM) for $24 \mathrm{~h}$. Western blotting was conducted using the antibodies indicated. (D) SGC7901/DDP cells were transfected with a CIP2A expression plasmid (CIP2A ${ }^{\mathrm{OE}}$ ), and then western blotting

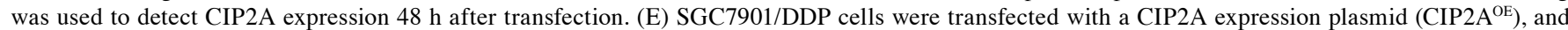
then trypan blue exclusion assay were used to detect proliferation, $48 \mathrm{~h}$ after transfection. (F) SGC7901/DDP cells were transfected with a CIP2A expression plasmid (CIP2 $\mathrm{A}^{\mathrm{OE}}$ ) for $48 \mathrm{~h}$.

of P-gp and HIF-1 $\alpha$ in SGC7901/DDP cells may in part contribute to the reversal of MDR. Considering that mTORC1 is a major negative regulator of autophagy (7), and $\mathrm{CuB}$ actives autophagy in SGC7901/DDP cells, we next determined whether $\mathrm{CuB}$ inhibited the activation of mTORC1. As shown in Fig. 4B, CuB decreased the phosphorylation of mTORC1 effectors (mTOR, p70S6K and 4E-BP1). Akt is an upstream regulator of $\mathrm{mTORC} 1$ and a downstream substrate of serine-threonine phosphatase PP2A (9). We tested effects of $\mathrm{CuB}$ on Akt phosphorylation (pAkt) and PP2A activity, and found that $\mathrm{CuB}$ downregulated pAkt and upregulated PP2A activity (Fig. 4C and D). These results suggested that CuB-induced mTORC1 inactivation may be mediated by PP2A/Akt. CIP2A is an oncogenic PP2A inhibitor protein that is highly expressed in malignant cancers including GC (31). Furthermore, we tested effects of $\mathrm{CuB}$ on CIP2A expression and found that the protein level of CIP2A decreased (Fig. 4E), indicating that $\mathrm{CuB}$ may target CIP2A to reactivate PP2A, then inhibits Akt/mTORC1 signal pathway.
CIP2A is essential for CuB-induced proliferation, autophagy, and apoptosis. We next examined whether CIP2A depletion would alter cellular sensitivity to $\mathrm{CuB}$. Two siRNAs targeting CIP2A were synthesized and used. As shown in Fig. 5A, knockdown by siRNA markedly decreased CIP2A protein in SGC7901/DDP cells. These data showed that CIP2A siRNA was specific and efficient in reducing CIP2A expression. To evaluate the role CIP2A plays in $\mathrm{CuB}$-induced proliferation inhibition, SGC7901/DDP cells were transfected with siRNA targeting CIP2A, followed by $\mathrm{CuB}$ treatment. Cell viability and western blotting were used to detect cell growth and protein expression. CIP2A depletion enhanced $\mathrm{CuB}$-induced growth inhibition (Fig. 5B), promoted CuB-induced autophagy and apoptosis effect (Fig. 5C). Of note, CIP2A depletion also enhanced $\mathrm{CuB}$-induced MDR inhibition. In addition, we examined whether an increase in CIP2A expression would alter cellular sensitivity to CuB. SGC7901/DDP cells were transfected with CIP2A plasmid and CIP2A expression was confirmed by western blotting (Fig. 5D). Notably, CIP2A 
overexpression antagonized $\mathrm{CuB}$-induced growth inhibition (Fig. 5E) and inhibited CuB-induced autophagy and apoptosis effects by upregulating pAkt and pmTOR (Fig. 5F). These data demonstrate that CIP2A plays a critical role in CuB-induced SGC7901/DDP autophagy and apoptosis.

\section{Discussion}

DDP, as a first-line chemotherapeutic drug for GC patients, especially for advanced stage, activated apoptosis by inducing DNA damage through crosslinking of the DNA (32). However, cancer cells often develop several mechanisms to overcome DDP-induced apoptosis and DNA damage, leading to DDP resistance (33-35). Therefore, investigation of the molecular mechanism conferring DDP resistance is urgently needed.

$\mathrm{CuB}$ is a representative natural compound for anticancer activities. Recently, $\mathrm{CuB}$ was reported to enhance the anticancer effects of DDP in lung, ovarian, cutaneous squamous cell carcinoma, and laryngeal squamous cell carcinoma (20,36-38). However, the relationship between $\mathrm{CuB}$ and DDP-resistance in GC cells has yet to be firmly established. In the present study, $\mathrm{CuB}$ alone showed an inhibitory effect on both DDP-sensitive and DDP-resistant GC cell lines (Fig. 2).

Apoptosis, which consists of extrinsic and intrinsic pathways, is the main cell death response to chemotherapy. Furthermore, evading apoptosis is one of the hallmarks of chemoresistance, and targeting apoptosis has become a cancer therapeutic strategy (39). Apoptosis is accompanied by various morphological changes, including nuclear condensation, DNA fragmentation, and apoptotic bodies. Nuclear morphology of SGC7901/DDP cells was analyzed using DAPI staining; significant nucleus condensation change in CuB treated SGC7901/ DDP was observed, which are typical characteristics of apoptosis (Fig. 3A). The extrinsic and intrinsic apoptotic pathways that ultimately lead to activation of effector casp-3 have been characterized (40,41). In SGC7901/DDP cells, treatment with $\mathrm{CuB}$ for $24 \mathrm{~h}$ caused downregulation of pro-casp-9 with generation of activated casp-9 (Fig. 3B). The intrinsic apoptotic signal led to activation of casp-3, reflected by a decrease of pro-casp-3 and increase of active casp-3 with cleavage of its substrate PARP (Fig. 3B). Thus, CuB may trigger apoptosis by activating the intrinsic apoptosis pathway which results in activation of effector casp-9. Autophagy is a programmed cell death that plays an important role in tumor progression and chemoresistance (42). In autophagy, LC3 II plays indispensable roles in autophagosome formation. Beclin1 functions as the key factor in the formation of autophagosomes (10). Our present results illustrated that $\mathrm{CuB}$ activated autophagy by the changes of LC3 II and Beclin 1 levels (Fig. 3C). In our further study, drug resistance is largely mediated through overexpression of MDR, HIF-1 $\alpha$, drug resistance protein, and proteasome subunits, increases in antioxidant defenses, and TOP2 activity; these results have been widely verified (33-35). The present study identified that the treatment of $\mathrm{CuB}$ was able to reverse the MDR of the SGC7901/DDP cells via the downregulation of P-gp, and HIF-1 $\alpha$ (Fig. 4A).

CIP2A, originally named KIAA1524 or P90, was cloned from patients with HCC (14). Induction of CIP2A is often associated with chemoresistance in cancer cells, and the inhibition of CIP2A in combination with chemotherapy may enhance the efficacy of cancer treatment (43). The functions of CIP2A in autophagy are not fully understood yet. Puustinen et al reported that the CIP2A/PP2A/mTORC1 signal axis is responsible for promoting cell growth and autophagy inhibition (7). They found that CIP2A can associate with mTORC1 and act as an allosteric inhibitor of mTORC1associated PP2A, thereby enhancing mTORC1-dependent growth signaling and inhibiting autophagy. Our present study demonstrated that $\mathrm{CuB}$ promoted the formation of autophagy via the inhibition of mTORC1 through dephosphorylating p70S6K and 4E-BP1 (Fig. 4B). Next, we detected CIP2A expression and $\mathrm{PP} 2 \mathrm{~A}$ activation and found that $\mathrm{CuB}$ significantly downregulated CIP2A expression and upregulated PP2A activity (Fig. 4C-E). Furthermore, we knocked down CIP2A expression in SGC7901/DDP cells and found that CIP2A depletion significantly promotes $\mathrm{CuB}$ induced apoptosis, autophagy, and reversed MDR (Fig. 5A-C). Moreover, our results showed that CIP2A overexpression significantly antagonized $\mathrm{CuB}$ induced cell proliferation, apoptosis and autophagy (Fig. 5D-F). Our data validated the mechanism by which CuB-induced cancer cell apoptosis and autophagy in SGC7901/DDP cells, that is, induction of cancer cell apoptosis and autophagy by inhibiting CIP2A to reactivate PP2A and enhance PP2A-dependent mTORC1 inactivation.

In conclusion, our results suggest that further studies on the detailed molecular modification of the CIP2A/PP2A/mTORC1 signaling axis by $\mathrm{CuB}$ and exploring its possible application in other malignant diseases are warranted.

\section{Acknowledgements}

This work was supported by grants from Open Ended Design Project from Hubei Province Key Laboratory of Conservation Biology for Shennongjia Golden Monkey (grant no. 2016SNJ001); the Natural Science Foundation of Hubei Province of China (grant no. 2016CFB528); the Foundation of Health and Family planning Commission of Hubei Province (grant no. WJ2017F067); the Foundation of Hubei University of Medicine (FDFR201605); the Foundation for Innovative Research Team of Hubei University of Medicine (2014CXX05); the Key Discipline Project of Hubei University of Medicine and the National Training Program of Innovation and Entrepreneurship for undergraduates (grant no. 201610929001).

\section{References}

1. Siegel RL, Miller KD and Jemal A: Cancer statistics, 2015. CA Cancer J Clin 65: 5-29, 2015.

2. Chen W, Zheng R, Baade PD, Zhang S, Zeng H, Bray F, Jemal A, Yu XQ and He J: Cancer statistics in China, 2015. CA Cancer J Clin 66: 115-132, 2016.

3. Yang X, Cai H, Liang Y, Chen L, Wang X, Si R, Qu K, Jiang Z, Ma B, Miao C, et al: Inhibition of c-Myc by let-7b mimic reverses mutidrug resistance in gastric cancer cells. Oncol Rep 33: 1723-1730, 2015.

4. Faneyte IF, Kristel PM, Maliepaard M, Scheffer GL, Scheper RJ, Schellens JH and van de Vijver MJ: Expression of the breast cancer resistance protein in breast cancer. Clin Cancer Res 8: 1068-1074, 2002.

5. Hong L, Piao Y, Han Y, Wang J, Zhang X, Du Y, Cao S, Qiao T, Chen $\mathrm{Z}$ and Fan D: Zinc ribbon domain-containing 1 (ZNRD1) mediates multidrug resistance of leukemia cells through regulation of P-glycoprotein and Bcl-2. Mol Cancer Ther 4: 1936-1942, 2005. 
6. Radisavljevic Z: AKT as locus of cancer multidrug resistance and fragility. J Cell Physiol 228: 671-674, 2013.

7. Puustinen P, Rytter A, Mortensen M, Kohonen P, Moreira JM and Jäättelä M: CIP2A oncoprotein controls cell growth and autophagy through mTORC1 activation. J Cell Biol 204: 713-727, 2014.

8. Demitrack ES, Gifford GB, Keeley TM, Carulli AJ, VanDussen KL, Thomas D, Giordano TJ, Liu Z, Kopan R and Samuelson LC: Notch signaling regulates gastric antral LGR5 stem cell function. EMBO J 34: 2522-2536, 2015.

9. Erazo T, Lorente M, López-Plana A, Muñoz-Guardiola P, Fernández-Nogueira P, García-Martínez JA, Bragado P, Fuster G, Salazar M, Espadaler J, et al: The new antitumor drug ABTL0812 inhibits the Akt/mTORC1 axis by upregulating Tribbles-3 pseudokinase. Clin Cancer Res 22: 2508-2519, 2016.

10. Jin HO, Hong SE, Park JA, Chang YH, Hong YJ, Park IC and Lee JK: Inhibition of JNK-mediated autophagy enhances NSCLC cell sensitivity to mTORC1/2 inhibitors. Sci Rep 6: 28945, 2016.

11. Agarwal S, Bell CM, Taylor SM and Moran RG: p53 deletion or hotspot mutations enhance mTORC1 activity by altering lysosomal dynamics of TSC2 and Rheb. Mol Cancer Res 14: 66-77, 2016.

12. Wu WD, Hu ZM, Shang MJ, Zhao DJ, Zhang CW, Hong DF and Huang DS: Cordycepin down-regulates multiple drug resistant (MDR)/HIF-1 $\alpha$ through regulating AMPK/mTORC1 signaling in GBC-SD gallbladder cancer cells. Int J Mol Sci 15 12778-12790, 2014.

13. Puustinen $P$ and Jäättelä M: KIAA1524/CIP2A promotes cancer growth by coordinating the activities of MTORC1 and MYC Autophagy 10: 1352-1354, 2014.

14. Junttila MR, Puustinen P, Niemelä M, Ahola R, Arnold H, Böttzauw T, Ala-aho R, Nielsen C, Ivaska J, Taya Y, et al: CIP2A inhibits PP2A in human malignancies. Cell 130: 51-62, 2007.

15. Li W, Ge Z, Liu C, Liu Z, Björkholm M, Jia J and Xu D: CIP2A is overexpressed in gastric cancer and its depletion leads to impaired clonogenicity, senescence, or differentiation of tumor cells. Clin Cancer Res 14: 3722-3728, 2008.

16. Liu Z, Ma L, Wen ZS, Hu Z, Wu FQ, Li W, Liu J and Zhou GB Cancerous inhibitor of PP2A is targeted by natural compound celastrol for degradation in non-small-cell lung cancer. Carcinogenesis 35: 905-914, 2014.

17. Ren J, Li W, Yan L, Jiao W, Tian S, Li D, Tang Y, Gu G, Liu H and $\mathrm{Xu} \mathrm{Z:} \mathrm{Expression} \mathrm{of} \mathrm{CIP2A} \mathrm{in} \mathrm{renal} \mathrm{cell} \mathrm{carcinomas} \mathrm{correlates}$ with tumour invasion, metastasis and patients' survival. Br J Cancer 105: 1905-1911, 2011

18. Liu CY, Shiau CW, Kuo HY, Huang HP, Chen MH, Tzeng CH and Chen KF: Cancerous inhibitor of protein phosphatase $2 \mathrm{~A}$ determines bortezomib-induced apoptosis in leukemia cells. Haematologica 98: 729-738, 2013

19. Zhang X, Xu B, Sun C, Wang L and Miao X: Knockdown of CIP2A sensitizes ovarian cancer cells to cisplatin: An in vitro study. Int J Clin Exp Med 8: 16941-16947, 2015.

20. El-Senduny FF, Badria FA, El-Waseef AM, Chauhan SC and Halaweish F: Approach for chemosensitization of cisplatinresistant ovarian cancer by Cucurbitacin B. Tumour Biol 37 : 685-698, 2016.

21. Chan KT, Meng FY, Li Q, Ho CY, Lam TS, To Y, Lee WH, Li M, Chu KH and Toh M: Cucurbitacin B induces apoptosis and S phase cell cycle arrest in BEL-7402 human hepatocellular carcinoma cells and is effective via oral administration. Cancer Lett 294: 118-124, 2010

22. Chan KT, Li K, Liu SL, Chu KH, Toh $M$ and Xie WD: Cucurbitacin B inhibits STAT3 and the Raf/MEK/ERK pathway in leukemia cell line K562. Cancer Lett 289: 46-52, 2010.

23. Liu Y, Cao W, Zhang B, Liu YQ, Wang ZY, Wu YP, Yu XJ, Zhang XD, Ming PH, Zhou GB, et al: The natural compound magnolol inhibits invasion and exhibits potential in human breast cancer therapy. Sci Rep 3: 3098, 2013.

24. Feng T, Cao W, Shen W, Zhang L, Gu X, Guo Y, Tsai H, Liu X, Li J,Zhang J, et al: Arctigenin inhibits STAT3 and exhibits anticancer potential in human triple-negative breast cancer therapy. Oncotarget 8: 329-344, 2017.
25. Cao W, Liu Y,Zhang R, Zhang B, Wang T, Zhu X, Mei L, Chen $\mathrm{H}$, Zhang H, Ming P, et al: Homoharringtonine induces apoptosis and inhibits STAT3 via IL-6/JAK1/STAT3 signal pathway in Gefitinib-resistant lung cancer cells. Sci Rep 5: 8477, 2015.

26. Chou CC, Yang JS, Lu HF, Ip SW, Lo C, Wu CC, Lin JP, Tang NY, Chung JG, Chou MJ, et al: Quercetin-mediated cell cycle arrest and apoptosis involving activation of a caspase cascade through the mitochondrial pathway in human breast cancer MCF-7 cells. Arch Pharm Res 33: 1181-1191, 2010.

27. Xiao X, He Z, Cao W, Cai F, Zhang L, Huang Q, Fan C, Duan C, Wang X, Wang $\mathrm{J}$, et al: Oridonin inhibits gefitinib-resistant lung cancer cells by suppressing EGFR/ERK/MMP-12 and CIP2A/ Akt signaling pathways. Int J Oncol 48: 2608-2618, 2016.

28. Cai F, Zhang L, Xiao X, Duan C, Huang Q, Fan C, Li J, Liu X, $\mathrm{Li} \mathrm{S}$ and Liu Y: Cucurbitacin B reverses multidrug resistance by targeting CIP2A to reactivate protein phosphatase 2A in MCF-7/ adriamycin cells. Oncol Rep 36: 1180-1186, 2016.

29. Qian HR and Yang Y: Functional role of autophagy in gastric cancer. Oncotarget 7: 17641-17651, 2016.

30. Lin L and Baehrecke EH: Autophagy, cell death, and cancer. Mol Cell Oncol 2: e985913-e985913, 2015.

31. Rincón R, Cristóbal I, Zazo S, Arpí O, Menéndez S, Manso R, Lluch A, Eroles P, Rovira A, Albanell J, et al: PP2A inhibition determines poor outcome and doxorubicin resistance in early breast cancer and its activation shows promising therapeutic effects. Oncotarget 6: 4299-4314, 2015.

32. Simonian PL, Grillot DA and Nuñez G: Bcl-2 and Bcl-XL can differentially block chemotherapy-induced cell death. Blood 90: 1208-1216, 1997.

33. Alcantara LM, Kim J, Moraes CB, Franco CH, Franzoi KD, Lee S, Freitas-Junior LH and Ayong LS: Chemosensitization potential of P-glycoprotein inhibitors in malaria parasites. Exp Parasitol 134: 235-243, 2013.

34. Luo L, Sun YJ, Yang L, Huang S and Wu YJ: Avermectin induces P-glycoprotein expression in $\mathrm{S} 2$ cells via the calcium/calmodulin/ NF-кB pathway. Chem Biol Interact 203: 430-439, 2013.

35. Liu Y, Zhu ZA, Liu QH, Kong QY, Liu L, Cui T and Wu YP: RAD001 can reverse drug resistance of SGC7901/DDP cells. Tumour Biol 35: 9171-9177, 2014.

36. Marostica LL, Silva IT, Kratz JM, Persich L, Geller FC, Lang KL, Caro MS, Durán FJ, Schenkel EP and Simões CM: Synergistic antiproliferative effects of a new Cucurbitacin B derivative and chemotherapy drugs on lung cancer cell line A549. Chem Res Toxicol 28: 1949-1960, 2015.

37. Chen W, Leiter A, Yin D, Meiring M, Louw VJ and Koeffler HP: Cucurbitacin B inhibits growth, arrests the cell cycle, and potentiates antiproliferative efficacy of cisplatin in cutaneous squamous cell carcinoma cell lines. Int J Oncol 37: 737-743, 2010.

38. Liu T, Peng H, Zhang M, Deng Y and Wu Z: Cucurbitacin B, a small molecule inhibitor of the Stat 3 signaling pathway, enhances the chemosensitivity of laryngeal squamous cell carcinoma cells to cisplatin. Eur J Pharmacol 641: 15-22, 2010.

39. Hanahan D and Weinberg RA: Hallmarks of cancer: The next generation. Cell 144: 646-674, 2011.

40. Nicholson DW: Caspase structure, proteolytic substrates, and function during apoptotic cell death. Cell Death Differ 6: 1028-1042, 1999 .

41. Johnstone RW, Ruefli AA and Lowe SW: Apoptosis: A link between cancer genetics and chemotherapy. Cell 108: 153-164, 2002.

42. Zhang M, Zhang H, Tang F, Wang Y, Mo Z, Lei X and Tang S: Doxorubicin resistance mediated by cytoplasmic macrophage colony-stimulating factor is associated with switch from apoptosis to autophagic cell death in MCF-7 breast cancer cells. Exp Biol Med (Maywood) 241: 2086-2093, 2016

43. Come C, Laine A, Chanrion M, Edgren H, Mattila E, Liu X, Jonkers J, Ivaska J, Isola J, Darbon JM, et al: CIP2A is associated with human breast cancer aggressivity. Clin Cancer Res 15: 5092-5100, 2009 . 